\title{
Inconsistency between the general theory of relativity and the experimental results of Faraday's unipolar inductor
}

\author{
Konstantinos Patrinos \\ National Technical University of Athens
}

\begin{abstract}
The non-inertiality of the rotating system of Faraday's unipolar generator forces us to address this problem using the principles of the general theory of relativity. The purpose of this study is to compare the theoretical quantitative estimates of induced electromotive force with the experimental results obtained from the corresponding measurements. The theoretical elaboration of this issue proves that the differences between the results of the general theory in relation to those of the special theory of relativity are some negligible terms, which are due to the non-inertiality of the rotating reference system. This result enables us to consider the theoretical estimates based on special and general relativity as equivalent, with quite satisfactory accuracy. Therefore, a very serious issue of inconsistency between the theory of relativity and the measurements of induced electromotive force emerges, since as already shown in the existing literature, this inconsistency, from the point of view of the special theory of relativity, is already proven.
\end{abstract}

Keywords - Faraday's homopolar generator, rotating reference system, electromagnetism in non-inertial systems, Faraday's experiment, Lorentz force in a rotating system

\section{Introduction}

The main part of the present work concerns the calculation of Lorentz force and electromotive force in a rotating reference system, based on the electrodynamics of the general theory of relativity. Starting from the principle of least action we calculate the force acting on an electron in the conduction band of the rotating conductor, due to the existence of the electromagnetic field in the rotating reference system. Also, we calculate the components of the electromagnetic tensor in terms of the rotating reference system. The resulting components of the electromagnetic force acting on the electron, and the developing electromotive force in the rotating conductor, are ultimately expressed as a function of the components of the electromagnetic field and the velocities which are measured in an inertial reference system. This inertial reference system is supposed to be the inertial frame of the laboratory.

We then use these relativistic expressions to calculate the induced electromotive force of the unipolar generator. The first experiment was conceived and implemented by Faraday in the mid-nineteenth century (1852) and the results of this experiment had as a consequence the revision of his previous views on how electromotive force $(E M F)$ is induced in a conductor, which moves in a magnetic field. Since then until today, a number of theoretical and experimental works regarding to this subject have been done, based on the electrodynamics of special and general theory of relativity $[1,2,3,4,5,6]$.

\section{Basic theory}

In this section we consider the force acting on a particle within a gravitational field in the presence of an electromagnetic field.

The action $\mathcal{S}$, for a particle with mass $m$ and charge $e$, within an electromagnetic field, having vector potential $A_{i}([7], \S 23$. The electromagnetic field tensor, $\S 87$. Motion of a particle in a gravitational field (PROBLEM), §90. The equations of electrodynamics in the presence of a gravitational field), is given by the following equation:

$$
\mathcal{S}=\int_{a}^{b}\left(-m c d s+\frac{e}{c} A_{i} d x^{i}\right)
$$

where $d s$, for a curvilinear coordinate system ([8], §5. Curved space), is given by:

$$
-d s^{2}=g_{i j} d x^{i} d x^{j}
$$


and $g_{i j}$ are covariant components of the metric tensor.

In equation (1.1) $c$ is the velocity of light in vacuum and $d x^{i}$ is the displacement element in the curvilinear system.

From equation (1.2) we obtain:

$$
\delta(-d s)=\frac{1}{2}\left(\delta g_{i j}\right) u^{i} d x^{j}+g_{i j} u^{i} d\left(\delta x^{j}\right)
$$

According to the principle of least action we have:

$$
\begin{aligned}
\delta \mathcal{S} & =m c \int_{a}^{b} \delta(-d s)+\frac{e}{c} \int_{a}^{b}\left\{\left(\delta A_{i}\right) d x^{i}+A_{i} \delta\left(d x^{i}\right)\right\} \\
& =m c \int_{a}^{b}\left\{-\Gamma_{k, i j} u^{i} u^{j}-g_{i k} \frac{d u^{i}}{d s}\right\} \delta x^{k} d s+\frac{e}{c} \int_{a}^{b} F_{k i} u^{i} \delta x^{k} d s=0
\end{aligned}
$$

where $u^{i}$ is the four-vector of velocity in the Curvilinear coordinate system and $\Gamma_{k, i j}$ Christoffel symbols.

Equation (1.4) reduces to:

$$
\frac{e}{c} F_{k i} u^{i}=m c\left\{\Gamma_{k, i j} u^{i} u^{j}+g_{i k} \frac{d u^{i}}{d s}\right\}
$$

where $F_{k i}$ is the electromagnetic tensor ([9], 5 ELECTRODYNAMICS IN VACUUM), that obeys the equation:

$$
\begin{aligned}
F_{k i} & =A_{k ; i}-A_{i ; k} \\
& =\frac{\partial A_{k}}{\partial x^{i}}-\Gamma_{k i}^{l} A_{l}-\left(\frac{\partial A_{i}}{\partial x^{k}}-\Gamma_{i k}^{l} A_{l}\right) \\
& =\frac{\partial A_{k}}{\partial x^{i}}-\frac{\partial A_{i}}{\partial x^{k}}
\end{aligned}
$$

Equation (1.5) can be written as :

$$
{ }_{-}^{e} F^{k i} u_{i}=m c\left\{\Gamma_{i j}^{k} u^{i} u^{j}+\frac{d u^{k}}{d s}\right\}
$$

Now, if each of the indices $\alpha, \beta, \gamma, \delta$ takes the values $1,2,3$, and $\gamma_{\alpha \beta}$ is the space metric tensor, which is related with the metric tensor $g_{\alpha \beta}$, according to the relation $\gamma_{\alpha \beta}=-g_{\alpha \beta}+h g_{\alpha} g_{\beta}$, where $h=g_{00}$, and $g_{\alpha}=-g_{0 \alpha} / g_{00}=-g_{0 \alpha} / h([7], \S 84$. Distances and time intervals), then the corresponding Christoffel symbols for the space will be:

$$
\lambda_{\beta \gamma}^{\alpha}=\frac{1}{2} \gamma^{\alpha \delta}\left(\frac{\partial \gamma_{\delta \beta}}{\partial x^{\gamma}}+\frac{\partial \gamma_{\delta \gamma}}{\partial x^{\beta}}-\frac{\partial \gamma_{\beta \gamma}}{\partial x^{\delta}}\right)
$$

We will also have:

$$
\begin{aligned}
\Gamma_{\beta \gamma}^{\alpha} & =\lambda_{\beta \gamma}^{\alpha}+\frac{h}{2}\left(g_{\beta}\left(g_{\gamma}{ }^{; \alpha}-g_{; \gamma}^{\alpha}\right)+g_{\gamma}\left(g_{\beta}{ }^{; \alpha}-g_{; \beta}^{\alpha}\right)\right)+\frac{1}{2} h^{; \alpha} g_{\beta} g_{\gamma} \\
\Gamma_{0 \beta}^{\alpha} & =\frac{h}{2}\left(g_{; \beta}^{\alpha}-g_{\beta}{ }^{; \alpha}\right)-\frac{1}{2} g_{\beta} h^{; \alpha} \\
\Gamma_{00}^{\alpha} & =\frac{1}{2} h^{; \alpha}
\end{aligned}
$$

From equations (1.8) and (1.9a)-(1.9c) we get:

$$
\Gamma_{i j}^{\alpha} u^{i} u^{j}=\frac{h^{; \alpha}}{2 h\left(1-\frac{v^{2}}{c^{2}}\right)}+\frac{\sqrt{h}\left(g_{; \beta}^{\alpha}-g_{\beta}^{; \alpha}\right) v^{\beta}}{c\left(1-\frac{v^{2}}{c^{2}}\right)}+\frac{\lambda_{\beta \gamma}^{\alpha} v^{\beta} v^{\gamma}}{c^{2}\left(1-\frac{v^{2}}{c^{2}}\right)}
$$

The total three dimensional force vector will be:

$$
f^{\alpha}=\frac{D P^{\alpha}}{d \tau}=c \sqrt{1-\frac{v^{2}}{c^{2}}} \frac{d}{d s} \frac{m v^{\alpha}}{\sqrt{1-\frac{v^{2}}{c^{2}}}}+\lambda_{\beta \gamma}^{\alpha} \frac{m v^{\beta} v^{\gamma}}{\sqrt{1-\frac{v^{2}}{c^{2}}}}
$$

where $d \tau=d s / c \sqrt{1-\frac{v^{2}}{c^{2}}}, P^{\alpha}=m c u^{\alpha}, u^{\alpha}=v^{\alpha} / c \sqrt{1-\frac{v^{2}}{c^{2}}}$ 
Taking into account eqs (1.7) and (1.10), eq (1.11) takes the form:

$$
f^{\alpha}=-\frac{m c^{2} h^{; \alpha}}{2 h \sqrt{1-\frac{v^{2}}{c^{2}}}}-\frac{m c \sqrt{h}}{\sqrt{1-\frac{v^{2}}{c^{2}}}}\left(g_{; \beta}^{\alpha}-g_{\beta}^{; \alpha}\right) v^{\beta}+e \sqrt{1-\frac{v^{2}}{c^{2}}} F^{\alpha k} u_{k}
$$

Also, eq (1.12) has the final form:

$$
f^{\alpha}=-\frac{m c^{2} \gamma^{\alpha \delta} h_{; \delta}}{2 h \sqrt{1-\frac{v^{2}}{c^{2}}}}-\frac{m c \sqrt{h}}{\sqrt{1-\frac{v^{2}}{c^{2}}}} \gamma^{\alpha \delta}\left(\frac{\partial g_{\delta}}{\partial x^{\beta}}-\frac{\partial g_{\beta}}{\partial x^{\delta}}\right) v^{\beta}+e \sqrt{1-\frac{v^{2}}{c^{2}}} F^{\alpha k} u_{k}
$$

with $-g^{\alpha \beta}=\gamma^{\alpha \beta}$, and $g^{\alpha}=\gamma^{\alpha \beta} g_{\beta}=-g^{0 \alpha}([7], \S 84$. Distances and time intervals).

\section{EMF and electromagnetic force.}

In this section the theoretical study of the operation of the unipolar generator focuses on the calculation of the induced electromotive force $(E M F)$ along an open path $A B$ in the rotating reference system $F$. Then, using appropriate transformations, we calculate the corresponding $E M F$, which is measured in inertial reference system of laboratory, provided that the measuring instruments are located in the laboratory. In order to calculate the induced $E M F$, the expression of the Lorentz force and the line-integral across the curve $A B$ is used.

\subsection{The spacetime metric tensor components}

We define a rest frame of reference, $S^{\prime}$, with coordinates $x^{\prime}, y^{\prime}, z^{\prime}, t^{\prime}$, whose axis $Z^{\prime}$ coincides with axis $Z$ of frame $F$.

The coordinate transformations between $S^{\prime}\left(x^{\prime}, y^{\prime}, z^{\prime}, t^{\prime}\right)$ and $F(x, y, z, t)$ ([10], CHAPTER I, Frames of reference, Coordinate systems, and Coordinate Transformations) are:

$$
\begin{aligned}
x & =x^{\prime} \cos \omega t^{\prime}+y^{\prime} \sin \omega t^{\prime} \\
y & =-x^{\prime} \sin \omega t^{\prime}+y^{\prime} \cos \omega t^{\prime} \\
z & =z^{\prime} \\
t & =t^{\prime}
\end{aligned}
$$

At this point we note that the metric tensor of spacetime for the rest reference frame, $S^{\prime},([7], \S 6$. Fourvectors) has the following components:

$$
\begin{aligned}
& g_{00}^{\prime}=1 \\
& g_{0 \alpha}^{\prime}=g_{\alpha 0}^{\prime}=0 \quad(\text { for } \quad \alpha=1,2,3) \\
& g_{\alpha \beta}^{\prime}=-\delta_{\alpha \beta}= \begin{cases}-1 & \text { if } \quad \alpha=\beta \\
0 & \text { otherwise }\end{cases}
\end{aligned}
$$

while the metric tensor of spacetime for the rotating frame $F$ will be obtained, in covariant or contravariant form ([7], §83. Curvilinear coordinates), from the equations:

$$
\begin{aligned}
g_{k l} & =\frac{\partial x^{\prime}}{\partial x^{k}} \frac{\partial x^{\prime j}}{\partial x^{l}} g_{i j}^{\prime} \\
g^{k l} & =\frac{\partial x^{k}}{\partial x^{\prime}} \frac{\partial x^{l}}{\partial x^{\prime j}} g^{\prime i j}
\end{aligned}
$$

Thus, using eq $(2.15 \mathrm{a})$ and $(2.15 \mathrm{~b})$ the metric tensor $g_{k l}$, has the following nonzero components:

$$
\begin{aligned}
& g_{00}=1-\frac{\omega^{2}}{c^{2}}\left(x^{2}+y^{2}\right) \\
& g_{11}=g_{22}=g_{33}=-1 \\
& g_{10}=g_{01}=\frac{\omega}{c} y \\
& g_{02}=g_{20}=-\frac{\omega}{c} x
\end{aligned}
$$


While, the nonzero components of the contravariant metric tensor will be:

$$
\begin{aligned}
& g^{00}=1 \\
& g^{01}=g^{10}=\frac{\omega}{c} y \\
& g^{02}=g^{20}=-\frac{\omega}{c} x \\
& g^{11}=-1+\frac{\omega^{2}}{c^{2}} y^{2} \\
& g^{12}=g^{21}=-\frac{\omega^{2}}{c^{2}} x y \\
& g^{22}=-1+\frac{\omega^{2}}{c^{2}} x^{2} \\
& g^{33}=-1
\end{aligned}
$$

\subsection{The total force in vector form, in rotating reference frame}

Now, we will consider the case of an electron with mass $m$ and charge $e$ as it is observed from a rotating reference frame, $F(x, y, z, t)$, with angular velocity $\omega$, in the presence of an electromagnetic field.

In order to elaborate on the topic of this subsection we need to consider the definitions of the vector product of two spatial vectors in a curvilinear coordinate system ([7], §88. The constant gravitational field (PROBLEMS, footnote)). So, we take into account the following definitions:

$$
\begin{aligned}
& C_{\alpha}=\frac{1}{2} \sqrt{\gamma} \epsilon_{\alpha \beta \gamma} C^{\beta \gamma}=\sqrt{\gamma} \epsilon_{\alpha \beta \gamma} A^{\beta} B^{\gamma} \\
& C^{\alpha}=\frac{1}{2 \sqrt{\gamma}} \epsilon^{\alpha \beta \gamma} C_{\beta \gamma}=\frac{1}{\sqrt{\gamma}} \epsilon^{\alpha \beta \gamma} A_{\beta} B_{\gamma}
\end{aligned}
$$

and vice versa,

$$
C_{\alpha \beta}=\sqrt{\gamma} \epsilon_{\alpha \beta \gamma} C^{\gamma}, \quad C^{\alpha \beta}=\frac{1}{\sqrt{\gamma}} \epsilon^{\alpha \beta \gamma} C_{\gamma}
$$

where $\mathbf{C}=\mathbf{A} \times \mathbf{B}$, and $\gamma$ is the determinant, formed from the quantities $\gamma_{\alpha \beta}$, and has the value $\gamma=1 / h$ for the above mentioned rotating reference frame $F$. Also, $\epsilon_{123}=\epsilon^{123}=1$, with the sign changing to interchanges of indicators. The rotation (curl) of a spatial vector function will be:

$$
(\operatorname{curl} \boldsymbol{A})^{\alpha}=\frac{1}{2 \sqrt{\gamma}} \epsilon^{\alpha \beta \gamma}\left(\frac{\partial A_{\gamma}}{\partial x^{\beta}}-\frac{\partial A_{\beta}}{\partial x^{\gamma}}\right)
$$

In the rotating reference system $F$, from the components of the metric tensor, we get the relation:

$$
\frac{\partial g_{2}}{\partial x^{1}}-\frac{\partial g_{1}}{\partial x^{2}}=\frac{2 \omega}{h^{2} c}
$$

According to the latter definitions eq. (2.18) takes the form:

$$
\nabla \times \boldsymbol{g}=\frac{2 \boldsymbol{\omega}}{h^{2} c}
$$

Also, in the rotating reference system $F$, for $\alpha=1,2$, we get the relation:

$$
\gamma^{\alpha \delta} h_{; \delta}=-2 \frac{\omega^{2}}{c^{2}} x^{\alpha}
$$

while for $\alpha=3$ we get $\gamma^{3 \delta} h_{; \delta}=0$, so the equation (1.13) takes the form:

$$
\boldsymbol{f}=-\frac{m}{h \sqrt{1-\frac{v^{2}}{c^{2}}}} \boldsymbol{\omega} \times(\boldsymbol{\omega} \times \boldsymbol{r})+\frac{m c \sqrt{h}}{\sqrt{1-\frac{v^{2}}{c^{2}}}} \boldsymbol{v} \times(\boldsymbol{\nabla} \times \boldsymbol{g})+\boldsymbol{f}_{(e . m .)}
$$


where $\boldsymbol{f}$ is the total force in vector form and $\boldsymbol{f}_{(e . m .)}$ is the electromagnetic force. Using the equation (2.19), equation (2.21) becomes:

$$
\boldsymbol{f}=\frac{1}{h^{2} \sqrt{1-\frac{v^{2}}{c^{2}}}}\{-m h \boldsymbol{\omega} \times(\boldsymbol{\omega} \times \boldsymbol{r})+2 m \sqrt{h} \boldsymbol{v} \times \boldsymbol{\omega}\}+\boldsymbol{f}_{(e . m .)}
$$

If we define $\boldsymbol{\Omega}=\sqrt{h} \boldsymbol{\omega}$, then the total force acting on the electron will be:

$$
\boldsymbol{f}=\frac{1}{h^{2} \sqrt{1-\frac{v^{2}}{c^{2}}}}\{-m \boldsymbol{\Omega} \times(\boldsymbol{\Omega} \times \boldsymbol{r})+2 m \boldsymbol{v} \times \boldsymbol{\Omega}\}+\boldsymbol{f}_{(e . m .)}
$$

It is obvious that for low velocities $\left(\frac{v^{2}}{c^{2}} \ll 1, \sqrt{h} \simeq 1\right)$ the first term inside the bracket, in eq. (2.23), is analogous to the centrifugal force, while the second term is analogous to the Coriolis force. Both of these are very small compared to the $\boldsymbol{f}_{(e . m .)}$, because of the very small mass of the electron and hence, in eq.(2.23), these can be ignored.

Taking into account the components of the velocity in the rotating reference frame $F([7], \S 88$. The constant gravitational field), given by the following equations:

$$
u^{\alpha}=\frac{v^{\alpha}}{c \sqrt{1-\frac{v^{2}}{c^{2}}}}, \quad u^{0}=\frac{1}{\sqrt{h} \sqrt{1-\frac{v^{2}}{c^{2}}}}+\frac{g_{\alpha} v^{\alpha}}{c \sqrt{1-\frac{v^{2}}{c^{2}}}}
$$

the electromagnetic force in tensor form ([7], §90. The equations of electrodynamics in the presence of a gravitational field), based on the equation (1.13), is given by the following equations:

$$
\begin{aligned}
f_{(e . m .)}^{\alpha} & =e \sqrt{1-\frac{v^{2}}{c^{2}}} F^{\alpha k} u_{k} \\
& =e \sqrt{1-\frac{v^{2}}{c^{2}}}\left(g_{00} u^{0}+g_{0 \beta} u^{\beta}\right) F^{\alpha 0}+\left(g_{\beta 0} u^{0}+g_{\beta \gamma} u^{\gamma}\right) F^{\alpha \beta} \\
& =\frac{e}{c}\left\{\frac{c}{\sqrt{h}} g_{00} F^{\alpha 0}+\left(\frac{c}{\sqrt{h}} g_{0 \beta}-\left(-g_{\beta \gamma}+\frac{g_{0 \beta} g_{0 \gamma}}{g_{00}}\right) v^{\gamma}\right) F^{\alpha \beta}\right\} \\
& =e \sqrt{h}\left(F^{\alpha 0}+g_{\beta} F^{\beta \alpha}\right)+\frac{e}{c} v_{\beta} F^{\beta \alpha}
\end{aligned}
$$

In order to express the electromagnetic force in vector form, we define $F^{\alpha 0}=\mathcal{E}^{\alpha}$ and $F^{\beta \alpha}=\mathcal{H}^{\alpha \beta}$. So, according to the above definitions for the vector product, the equations $g_{\beta} F^{\beta \alpha}=g_{\beta} \mathcal{H}^{\alpha \beta}=\frac{1}{\sqrt{\gamma}} \epsilon^{\alpha \beta \gamma} g_{\beta} \mathcal{H}_{\gamma}$, $v_{\beta} F^{\beta \alpha}=\frac{1}{\sqrt{\gamma}} \epsilon^{\alpha \beta \gamma} v_{\beta} \mathcal{H}_{\gamma}$ are taken, and the relation for the electromagnetic force will be:

$$
\boldsymbol{f}_{(e . m .)}=e \sqrt{h}(\mathcal{E}+\boldsymbol{g} \times \mathcal{H})+\frac{e}{c} \boldsymbol{v} \times \mathcal{H}
$$

The equation (2.25) gives the Lorentz force in the rotating reference frame $F$. The electromotive force $(E M F)$ along an open path $A B$ in the frame $F$ is given by the following relation:

$$
\begin{aligned}
E M F & =\frac{1}{e} \int_{A}^{B} \gamma_{\alpha \beta} f_{(e . m .)}^{\alpha} d x^{\beta} \\
& =\int_{A}^{B}\left\{\sqrt{h} \mathcal{E}^{\alpha}+\sqrt{h}(\boldsymbol{g} \times \mathcal{H})^{\alpha}+\frac{1}{c}(\boldsymbol{v} \times \mathcal{H})^{\alpha}\right\} \gamma_{\alpha \beta} d x^{\beta}
\end{aligned}
$$

where $(\boldsymbol{g} \times \mathcal{H})^{\alpha}$ and $(\boldsymbol{v} \times \mathcal{H})^{\alpha}$ are the $\alpha$-components of the vector products $\boldsymbol{g} \times \boldsymbol{H}$ and $\boldsymbol{v} \times \mathcal{H}$ respectively, and $\boldsymbol{v}$ is the velocity of the electron in the rotating reference system $F$. However, in this case the Lorentz force and the $E M F$ are expressed as a function of $\mathcal{E}$ and $\mathcal{H}$ which are measured in the reference system $F$, while these quantities should be expressed as a function of electric and magnetic field in an inertial reference system, given that these values are directly measurable in an inertial system. Driven by this fact we will then calculate the components of the electromagnetic tensor of the rotating reference system, as a function of the values of the electric and magnetic field in the inertial reference system. We will also calculate the correlation of velocities between the rotating and the inertial reference system. 


\subsection{The electromagnetic tensor}

We will examine then the formulas that relate the covariant and contravariant components of the electromagnetic tensor in the rotating frame, to the components of the electromagnetic field in the inertial frame.

If ${F^{\prime}}^{i j}$ is the contravariant electromagnetic tensor, in the inertial frame $S^{\prime}([7], \S 23$. The electromagnetic field tensor), and $F^{k l}$ is the corresponding tensor in the rotating frame, then they will transform according to the relation:

$$
F^{k l}=\frac{\partial x^{k}}{\partial x^{\prime}} \frac{\partial x^{l}}{\partial x^{\prime j}} F^{\prime i j}
$$

Considering that ${F^{\prime}}^{10}=E_{x}^{\prime},{F^{\prime}}^{20}=E_{y}^{\prime},{F^{\prime}}^{30}=E_{z}^{\prime}$, and using the equations (2.14a)-(2.14d) we find:

$$
\begin{aligned}
& F^{10}=E_{x}^{\prime} \cos \omega t+E_{y}^{\prime} \sin \omega t \\
& F^{20}=-E_{x}^{\prime} \sin \omega t+E_{y}^{\prime} \cos \omega t \\
& F^{30}=E_{z}^{\prime}
\end{aligned}
$$

Now, at this point, we will define the inertial reference frame $S$. The inertial frame $S$, which is at rest, is rotated at an angle $\omega t$, with respect to the inertial frame $S^{\prime}$. Momentarily, in $S^{\prime}$, all axis of the frames $S$ and $F$ will coincide. If $E_{x}, E_{y}, E_{z}$ are the components of the electric field in $S$, then they are related to the components $E_{x}^{\prime}, E_{y}^{\prime}, E_{z}^{\prime}$ according to the relation:

$$
\left(\begin{array}{l}
E_{x} \\
E_{y} \\
E_{z}
\end{array}\right)=\left(\begin{array}{ccc}
\cos \omega t & \sin \omega t & 0 \\
-\sin \omega t & \cos \omega t & 0 \\
0 & 0 & 1
\end{array}\right)\left(\begin{array}{c}
E_{x}^{\prime} \\
E_{y}^{\prime} \\
E_{z}^{\prime}
\end{array}\right)
$$

From the comparison of the eq. (2.29) with the relations $(2.28 \mathrm{a})-(2.28 \mathrm{c})$, it is concluded that the components $F^{10}, F^{20}, F^{30}$ of the $F^{k l}$ tensor of the rotating frame $F$, are equal to the components of the electric field in the frame $S$. Next we define the components $H_{x}, H_{y}, H_{z}$ of the magnetic field in the inertial reference system $S$ as follows:

$$
\begin{aligned}
& H_{x}=H_{x}^{\prime} \cos \omega t+H_{y}^{\prime} \sin \omega t \\
& H_{y}=-H_{x}^{\prime} \sin \omega t+H_{y}^{\prime} \cos \omega t \\
& H_{z}=H_{z}^{\prime}
\end{aligned}
$$

Also given that ${F^{\prime}}^{32}=H^{\prime}{ }_{x},{F^{\prime}}^{13}=H^{\prime}{ }_{y},{F^{\prime 2}}^{21}=H^{\prime}{ }_{z}$ and based on the equations (2.27) and (2.30a)-(2.30c) we get the following relations:

$$
\begin{aligned}
F^{21} & =\frac{\omega}{c} x E_{x}+H_{z} \\
F^{13} & =-\frac{\omega}{c} y E_{z}+H_{y} \\
F^{32} & =-\frac{\omega}{c} x E_{z}+H_{x}
\end{aligned}
$$

Similarly, from the transformation equation of the components of the covariant electromagnetic tensor, which is expressed as follows:

$$
F_{k l}=\frac{\partial x^{\prime}}{\partial x^{k}} \frac{\partial x^{\prime j}}{\partial x^{l}} F_{i j}^{\prime}
$$

since $H_{z}^{\prime}=F_{21}^{\prime}, H_{y}^{\prime}=F_{13}^{\prime}, H_{x}^{\prime}=F_{32}^{\prime}$ and $E_{x}^{\prime}=F_{01}^{\prime}, E_{y}^{\prime}=F_{02}^{\prime}, E_{z}^{\prime}=F_{03}^{\prime}$, according to the equations (2.28a)-(2.28c) and (2.30a)-(2.30c) we get the following relations:

$$
\begin{aligned}
& F_{01}=E_{x}+\frac{\omega}{c} x H_{z} \\
& F_{02}=E_{y}+\frac{\omega}{c} y H_{z} \\
& F_{03}=E_{z}-\frac{\omega}{c}\left(x H_{x}+y H_{y}\right)
\end{aligned}
$$

The coordinates $x, y, z$, in the two reference systems $S$ and $F$ coincide momentarily. The second members of the equations (2.33a)-(2.33c) are expressions of the components of the electric and magnetic field of inertial frame $S$. With the help of the transformation relation (2.32), we get the relations:

$$
\begin{aligned}
& F_{21}=H_{z}^{\prime} \\
& F_{13}=-H_{x}^{\prime} \sin \omega t+H_{y}^{\prime} \cos \omega t \\
& F_{32}=H_{x}^{\prime} \cos \omega t+H^{\prime} y \sin \omega t
\end{aligned}
$$


and therefore:

$$
\left(\begin{array}{l}
F_{32} \\
F_{13} \\
F_{21}
\end{array}\right)=\left(\begin{array}{ccc}
\cos \omega t & \sin \omega t & 0 \\
-\sin \omega t & \cos \omega t & 0 \\
0 & 0 & 1
\end{array}\right)\left(\begin{array}{c}
H_{x}^{\prime} \\
H_{y}^{\prime} \\
H_{z}^{\prime}
\end{array}\right)
$$

From the equation (2.35) and the definition of the components of the magnetic field in the inertial reference system $S$ the equations $H_{x}=F_{32}, H_{y}=F_{13}, H_{z}=F_{21}$ are obtained. Therefore the components $F_{32}, F_{13}$, $F_{21}$ of the electromagnetic tensor $F_{k l}$, in the rotating reference system $F$, are equal to the components of the magnetic field which are measured in the inertial frame $S$.

\subsection{The velocity transformations}

In the following we will examine the velocity transformating equations, between frames $F$ and $S^{\prime}$. We have:

$$
u^{\alpha}=\frac{\partial x^{\alpha}}{\partial x^{\prime j}} u^{\prime j}
$$

If we define $u^{\prime \alpha}=\mathrm{g} v^{\prime \alpha} / c, u^{\prime 0}=\mathrm{g}$ and $\mathrm{g}=\left(1-v^{\prime 2} / c^{2}\right)^{-1 / 2}, \quad(\alpha=1,2,3),([7], \S 7$. Four-dimensional velocity), we obtain:

$$
\begin{aligned}
u^{1} & =\frac{1}{c}\left(\omega y+v_{x}^{\prime} \cos \omega t+v_{y}^{\prime} \sin \omega t\right) \mathrm{g} \\
u^{2} & =\frac{1}{c}\left(-\omega x-v_{x}^{\prime} \sin \omega t+v_{y}^{\prime} \cos \omega t\right) \mathrm{g} \\
u^{3} & =\frac{1}{c} v_{z}^{\prime} \mathrm{g}
\end{aligned}
$$
2.3:

Taking $V_{x}, V_{y}, V_{z}$, as the components of the velocity in $S$, we will obtain, using the analogy with subsection

$$
\left(\begin{array}{l}
V_{x} \\
V_{y} \\
V_{z}
\end{array}\right)=\left(\begin{array}{ccc}
\cos \omega t & \sin \omega t & 0 \\
-\sin \omega t & \cos \omega t & 0 \\
0 & 0 & 1
\end{array}\right)\left(\begin{array}{c}
v_{x}^{\prime} \\
v_{y}^{\prime} \\
v_{z}^{\prime}
\end{array}\right)
$$

Thus, from eqs (2.37a)-(2.37c) and (2.38) we find:

$$
\begin{aligned}
u^{1} & =\frac{1}{c}\left(\omega y+V_{x}\right) \mathrm{g} \\
u^{2} & =\frac{1}{c}\left(-\omega x+V_{y}\right) \mathrm{g} \\
u^{3} & =\frac{1}{c} V_{z} \mathrm{~g}
\end{aligned}
$$

Taking into account that $V^{2}=V_{x}^{2}+V_{y}^{2}+V_{z}^{2}={v_{x}^{\prime}}^{2}+{v_{y}^{\prime}}^{2}+{v_{z}^{\prime}}^{2}$, we have $\mathrm{g}=\left(1-{v^{\prime}}^{2} / c^{2}\right)^{-1 / 2}=$ $\left(1-V^{2} / c^{2}\right)^{-1 / 2}$. Also, in frame $F$ (for $\left.\alpha=1,2,3\right),([7], \S 88$. The constant gravitational field), we have:

$$
u^{\alpha}=\frac{v^{\alpha}}{c \sqrt{1-\frac{v^{2}}{c^{2}}}}
$$

Now, with the help of eqs (2.39a)-(2.39c) and (2.40) we obtain

$$
\begin{aligned}
& \frac{v_{x}}{\sqrt{1-\frac{v^{2}}{c^{2}}}}=\mathrm{g}\left(\omega y+V_{x}\right) \\
& \frac{v_{y}}{\sqrt{1-\frac{v^{2}}{c^{2}}}}=\mathrm{g}\left(-\omega x+V_{y}\right) \\
& \frac{v_{z}}{\sqrt{1-\frac{v^{2}}{c^{2}}}}=\mathrm{g} V_{z}
\end{aligned}
$$

where $v_{x}, v_{y}, v_{z}$ are the components of the velocity in frame $F$. 


\section{Calculation of the electromotive force}

At this point we will express the electromagnetic force, $\boldsymbol{f}_{(e . m .)}$, acting on an electron with charge $e$ and coordinates $x, y, z$, in frame $F$, as a function of the components of electric and magnetic fields, and the velocity $\boldsymbol{V}$ in $S$.

Considering that this elementary charge, $e$, is an electron in the conduction band of the rotating conductor, due to the very low velocity of the electrical current produced by the induced $E M F$, this electron can be considered as practically stationary in frame $F$. Under these conditions, the relation (2.24) for the electromagnetic force, becomes:

$$
f_{(e . m .)}^{\alpha}=e \sqrt{h}\left(F^{\alpha 0}+g_{\beta} F^{\beta \alpha}\right)
$$

and based on the calculations in subsection 2.3 the corresponding vector expression is:

$$
\begin{aligned}
\boldsymbol{f}_{(e . m .)}= & e \sqrt{h}\left\{\boldsymbol{E}+\frac{\omega}{h c}\left(x H_{z} \boldsymbol{i}+y H_{z} \boldsymbol{j}-\left(x H_{x}+y H_{y}\right) \boldsymbol{k}\right)\right. \\
& \left.+\frac{\omega^{2}}{h c^{2}}\left(x^{2} E_{x} \boldsymbol{i}+x y E_{x} \boldsymbol{j}+\left(x^{2}+y^{2}\right) E_{z} \boldsymbol{k}\right)\right\} \\
= & \frac{e}{\sqrt{h}}\left\{\boldsymbol{E}+\frac{\omega}{c}\left(x H_{z} \boldsymbol{i}+y H_{z} \boldsymbol{j}-\left(x H_{x}+y H_{y}\right) \boldsymbol{k}\right)\right. \\
& \left.-\frac{\omega^{2}}{c^{2}}\left(y^{2} E_{x} \boldsymbol{i}+\left(x y E_{x}+\left(x^{2}+y^{2}\right) E_{y}\right) \boldsymbol{j}\right)\right\}
\end{aligned}
$$

Since the velocity $v$ in the rotating reference system $F$ was considered zero, based on the equations (2.41a)(2.41c), and $v_{x}=v_{y}=v_{z}=0$, the velocity of the electron in inertial frame $\mathrm{S}$, in vector form, is:

$$
\boldsymbol{V}=-\omega y \boldsymbol{i}+\omega x \boldsymbol{j}
$$

Also if $g_{1}, g_{2}, g_{3}$ are the components of the vector $\boldsymbol{g}$, where $g_{1}=-g_{01} / g_{00}=-\omega y /(h c), g_{2}=-g_{02} / g_{00}=$ $\omega x /(h c), g_{3}=-g_{03} / g_{00}=0$ ([7], §84. Distances and time intervals), we get the equation $h \boldsymbol{g}=\boldsymbol{V} / c$.

The vector product of the velocity $\boldsymbol{V}$ and the magnetic field $\boldsymbol{H}$ in the inertial reference system $S$, is:

$$
\boldsymbol{V} \times \boldsymbol{H}=\omega\left(x H_{z} \boldsymbol{i}+y H_{z} \boldsymbol{j}-\left(x H_{x}+y H_{y}\right) \boldsymbol{k}\right)
$$

and therefore the equation (3.43) takes the following form:

$$
\boldsymbol{f}_{(e . m .)}=\frac{e}{\sqrt{h}}\left\{\boldsymbol{E}+\frac{1}{c} \boldsymbol{V} \times \boldsymbol{H}-\frac{\omega^{2}}{c^{2}}\left(y^{2} E_{x} i+\left(x y E_{x}+\left(x^{2}+y^{2}\right) E_{y}\right) \boldsymbol{j}\right)\right\}
$$

The factor $\mathrm{g}=\left(1-V^{2} / c^{2}\right)^{-1 / 2}$ is equal to the quantity $1 / \sqrt{h}$, since $h=g_{00}=1-\omega^{2}\left(x^{2}+y^{2}\right) / c^{2}=1-V^{2} / c^{2}$. Because the speed of rotation of even the farthest points of the rotating conductor from its axis of rotation is certainly very low compared to the speed of light in vacuum $(V \ll c, \omega x \ll c, \omega y \ll c)$, the factor $\mathrm{g}$ becomes approximately equal to the unit $(1 / \sqrt{h} \simeq 1)$ and the term $-\frac{\omega^{2}}{c^{2}}\left(y^{2} E_{x} i+\left(x y E_{x}+\left(x^{2}+y^{2}\right) E_{y}\right) \boldsymbol{j}\right)$ in the last equation can be ignored. Under these conditions the electromagnetic force is recalculated as follows:

$$
\boldsymbol{f}_{(e . m .)}=e \boldsymbol{E}+\frac{e}{c} \boldsymbol{V} \times \boldsymbol{H}
$$

Let us now define an inertial reference system $S^{\prime \prime}$ moving with velocity $\boldsymbol{V}$ with respect to the inertial reference system $S$, which is momentarily stationary with respect to the aforementioned electron. The electric field $\boldsymbol{E}^{\prime \prime}$ in this reference system will be determined by the known transformation relations of the electromagnetic field from one inertial reference system to another. For low velocities (as here in our case $V \ll c$, [7], §24. Lorentz transformation of the field) the electric field $\boldsymbol{E}^{\prime \prime}$ will be calculated according to the equation:

$$
\boldsymbol{E}^{\prime \prime}=\boldsymbol{E}+\frac{1}{c} \boldsymbol{V} \times \boldsymbol{H}
$$

The Lorentz force observed in the inertial system $S^{\prime \prime}$, is equal to the calculated electromagnetic force $\boldsymbol{f}_{(e . m .)}$ in the rotating reference system $F$. More precisely, any difference (on a theoretical level and not on a practical level of experimental measurement) between the two estimates of electromagnetic force in the inertial and noninertial (rotating) reference system, is determined by the previous negligible terms, whose existence is due to non-inertiality of the rotating frame, according to the general theory of relativity. 
The induced $E M F$, along an open path $A B$ in frame $F$, is given by the equation (2.26). The non-zero components of the three-dimensional metric tensor $\gamma_{\alpha \beta}$, with $\alpha, \beta=1,2,3$, according to the relation $\gamma_{\alpha \beta}=$ $-g_{\alpha \beta}+g_{0 \alpha} g_{0 \beta} / g_{00}$, are:

$$
\begin{aligned}
\gamma_{11} & =\frac{1}{h}\left(1-\frac{\omega^{2} x^{2}}{c^{2}}\right) \\
\gamma_{22} & =\frac{1}{h}\left(1-\frac{\omega^{2} y^{2}}{c^{2}}\right) \\
\gamma_{33} & =1 \\
\gamma_{21} & =\gamma_{12}=-\frac{1}{h} \frac{\omega^{2} x y}{c^{2}}
\end{aligned}
$$

For low speeds $(\omega x \ll c, \omega y \ll c)$ the components of the metric tensor $\gamma_{\alpha \beta}$ can be formulated as follows:

$$
\gamma_{\alpha \beta}=\delta_{\alpha \beta}= \begin{cases}1 & \text { if } \alpha=\beta \\ 0 & \text { otherwise }\end{cases}
$$

As we can easily see, in the equation (3.50), these are the components of the metric tensor of Euclidean space. Since under these conditions the space of the rotating reference system, in a very good approach, is forming a Euclidean space, the induced $E M F$ can be calculated according to the relation:

$$
E M F=\int_{A}^{B}\left(\boldsymbol{E}+\frac{1}{c} \boldsymbol{V} \times \boldsymbol{H}\right) \cdot d \boldsymbol{\ell}
$$

The expression for the electromotive force of the relation (3.51) is that of the special theory of relativity. But in Müller's excellent experimental research (in [6]), which deals with the experimental results of $E M F$ measurements of rotating conductors and magnets, it is clear that the special theory of relativity is not consistent with these results. Specifically (in [6], VIII. CONCLUSIONS) it refers:

"5) Finally, Special Relativity is applicable to the rectilinearly translating magnet, where the $-\mathbf{v} \times \mathbf{B}$ resulting from the Lorentz transformation of the fields, cancels the $+\mathbf{v} \times \mathbf{B}$ of the Lorentz force, yielding a net zero result as expected when magnet and wire co-move together. In rotation, however, the Special Theory cannot be used because it would yield always a zero emf contrary to observation. Being a local field theory, (as all field theories are), Special Relativity cannot account for the difference between a translating magnet with edges moving perpendicularly to themselves, and a rotating cylindrical magnet in which all edges move tangentially to themselves. The edge effect, being "far away" from the location where the conductor IR is, does not enter at all in any of the (local) field transformation equations of Special Relativity.

For rotation, therefore, the General Theory must be used. But this approach is out of the scope of this paper, and it can be evaded by using, instead, the more "down-to-earth" edge effect theory."

However, because in practice, as can be seen from the theoretical elaboration of this issue in the present work, the calculated $E M F$ based on the general theory of relativity is ultimately what is expected by making the calculation based on the special theory of relativity, a very serious issue of discrepancy emerges between the theoretical and experimental results concerning Faraday's unipolar inductor.

\section{Conclusions}

All the conclusions drawn from the present theoretical elaboration are summarized in the commentary of an only one relation and this is the relation (3.46). As we have seen all the quantities due to the non-inertiality of the rotating frame are negligible. The omission of these terms differentiates the theoretical result of the calculation of the electromotive force by a negligible amount, much smaller than the smallest possible experimental error that may be involved in the measurement of the electromotive force.

Due to this fact the resulting relation for the electromagnetic force, and consequently also for the electromotive force is, in a very good approach, that which arises from the special theory of relativity. This is evident from the beginning of the theoretical elaboration of this whole subject and specifically from an observation concerning the relations $(2.16 \mathrm{a})-(2.16 \mathrm{~d})$ and $(2.17 \mathrm{a})-(2.17 \mathrm{~g})$, which are represent the components of the metric tensor of a rotating reference system. Due to the low rotation speeds, compared to the speed of light in vacuum, the components of said metric tensor converge to the corresponding values of the components of the metric tensor of the inertial reference system, which are those of Minkowski's spacetime and of special theory of relativity.

The general conclusion is therefore that the Lorentz force and the electromotive force in a rotating frame, calculated on the basis of the theory of relativity, give theoretical results that are not consistent with the corresponding measurements on the Faraday's homopolar inductor. 


\section{References}

[1] P. J. Scanlon,R. N. Henriksen, and J. R. Allen "Approaches to Electromagnetic Induction" Am. J. Phys. 37 698-708 (1969)

[2] M. J. Crooks, D. B. Litvin, P. W. Matthews, R. Macaulay, J. Shaw "One-piece Faraday generator: A paradoxical experiment from 1851" Am. J. Phys. 46 729-731 (1978)

[3] ARTHUR I. MILLER "Unipolar Induction: a Case Study of the Interaction between Science and Technology" ANNALS OF SCIENCE, 38 (1981), 155-189

[4] Horace W. Crater "General covariance, Lorentz covariance, the Lorentz force, and the Maxwell equations" Am. J. Phys. 62 923-931 (1994)

[5] Jorge Guala-Valverde, Pedro Mazzoni, and Ricardo Achilles "The homopolar motor: A true relativistic engine" American Journal of Physics 70, 1052 (2002); doi: 10.1119/1.1498857

[6] Francisco J. Müller "Unipolar Induction Revisited: New Experiments and the "Edge Effect" Theory" IEEE TRANSACTIONS ON MAGNETICS, VOL. 50, NO. 1, JANUARY 2014

[7] L. Landau and E. Lifshitz, The Classical Theory of Fields Pergamon Press Ltd. (Copyright 1975)

[8] P. A. M. DIRAC, GENERAL THEORY OF RELATIVITY, John Wiley \& Sons (Copyright 1975)

[9] C. MØLLER, The Theory of Relativity, Oxford university Press, (Copyright 1972)

[10] P. G. BERGMANN, INTRODUCTION TO THE THEORY OF RELATIVITY, PRENTICE-HALL, INC. (Copyright 1942) 